\title{
Contemporary Urologic Minilaparoscopy: Indications, Techniques, and Surgical Outcomes in a Multi-institutional European Cohort
}

\author{
Francesco Porpiglia, MD, Riccardo Autorino, MD, PhD, FEBU, ${ }^{2,3}$ Antonio Cicione, ${ }^{4,5}$ \\ Vincenzo Pagliarulo, Mario Falsaperla, ${ }^{7}$ Alessandro Volpe, Ali Serdar Gozen, ${ }^{9}$ Antonio Celia, ${ }^{10}$ \\ Marco De Sio, ${ }^{2}$ Alberto Saita, ${ }^{7}$ Rocco Damiano, ${ }^{4}$ Monica Zacchero, ${ }^{8}$ Cristian Fiori, ${ }^{1}$ Carlo Terrone, \\ Riccardo Bertolo, Francesco Greco," Alberto Breda, ${ }^{12}$ Estevao Lima, MD, PhD, ${ }^{5}$ and Jens Rassweiler, MD ${ }^{9}$
}

\begin{abstract}
Objectives: To provide an analytical overview of contemporary indications, techniques, and outcomes of urologic minilaparoscopy (ML) in multiple European centers.

Methods: Data of patients who had undergone a minilaparoscopic urologic procedure at nine European institutions between 2009 and 2012 were retrospectively gathered. Surgical procedures were classified as upper or lower urinary tract and as ablative or extirpative and reconstructive. The main surgical outcome parameters were analyzed and relevant operative data related to the surgical technique were recorded.

Results: Overall, 192 patients (mean age 45.25 17.8 years) were included in the analysis. Most of them were nonobese (mean body mass index [BMI] $24.7 \pm 3.6 \mathrm{~kg} / \mathrm{m}^{2}$ ) at low estimated surgical risk (mean American Society of Anesthesiologists [ASA] 1.69 \pm 0.68 ). Indications for surgery were mostly nononcologic (132 cases, 68.8\%). Most of the procedures were done in the upper urinary tract $(133$ cases, $69.2 \%)$ and were mostly with a reconstructive intent (109 cases, 56.7\%). Overall operative time was $132.7 \pm 52.3$ minutes with an estimated blood loss of $60.9 \pm 47.6 \mathrm{~mL}$ while the mean hospital stay was $5 \pm 2.1$ days. Most of the postoperative complications were low Clavien grade ( 1 and 2$)$, with only one $(0.5 \%)$ grade 3 and one $(0.5 \%)$ grade 4 complications recorded. Conclusions: A broad range of common procedures can be safely and effectively performed with ML techniques. By duplicating the principles of standard laparoscopy, but potentially offering less surgical scar and trauma, ML can be regarded as a viable option when looking for a virtually "scarless" surgery.
\end{abstract}

\section{Introduction}

$\mathbf{T}$ HE IDEA of performing surgical procedures with no scar has gained attention in the urological community over the last 5 years. Based on this concept, novel surgical approaches, such as natural orifice translumenal endoscopic surgery (NOTES) and laparoendoscopic single-site surgery (LESS), have been explored with the ultimate aim of minimizing the surgical morbidity and fostering patient recovery. ${ }^{1}$
At this time, only hybrid NOTES or NOTES-assisted laparoscopic techniques seem to be ready for immediate implementation in clinical practice. ${ }^{2,3}$ LESS has been increasingly adopted worldwide, in urology and in other surgical specialties. ${ }^{4,5}$ Nevertheless, the claimed advantages of LESS over the traditional laparoscopic approach remain to be fully demonstrated and its disadvantages are widely recognized. ${ }^{1}$ Moreover, although feasible, pure LESS remains a technical challenge for the surgeon, so

${ }^{1}$ Department of Urology, San Luigi Gonzaga Hospital, Orbassano, Italy.

${ }^{2}$ Urology Unit, Second University of Naples, Naples, Italy.

${ }^{3}$ Center for Laparoscopic and Robotic Surgery, Glickman Urological and Kidney Institute, Cleveland Clinic, Cleveland, Ohio.

${ }^{4}$ Department of Urology, Magna Graecia University, Catanzaro, Italy.

${ }^{5}$ Department of Urology, Braga Hospital, Braga, Portugal.

${ }^{6}$ Department of Urology, Aldo Moro University, Bari, Italy.

${ }^{7}$ Department of Urology, Policlinico-Vittorio Emanuele, Catania, Italy.

${ }^{8}$ Department of Urology, University of Eastern Piedmont, Maggiore Della Carità Hospital, Novara, Italy.

${ }^{9}$ Department of Urology, SLK Kliniken Heilbronn, Heilbronn, Germany.

${ }^{10}$ Department of Urology, San Bassiano Hospital, Bassano Del Grappa, Italy.

${ }^{11}$ Department of Urology and Minimal Invasive Surgery, Romolo Hospital, Crotone, Italy.

${ }^{12}$ Department of Urology, Autonoma University of Barcelona, Fundació Puigvert, Barcelona, Spain. 
that a mix of techniques could be the key for its implementation. $^{6}$

Recently, minilaparoscopy (ML) has been rediscovered in an attempt to reduce the trauma on the abdominal wall derived from standard laparoscopic access, improving cosmetic outcome and recovery. ${ }^{7}$ This rediscovery has been fuelled by the availability of more reliable instrumentation and by the fact that ML allows minimal abdominal scar, meanwhile preserving the key principle of triangulation.

Over the last few years, many minilaparoscopic procedures have been successfully performed in several surgical disciplines. ${ }^{8,9}$ In urology, evidence supporting ML has been limited to small case series or case-control studies from selected centers with laparoscopic expertize. ${ }^{10-12}$ Thus, more robust analyses of larger samples are desirable to corroborate positive findings from early series.

This study was initiated as a collaborative effort among institutions pioneering the development of urologic ML, with the purpose of providing an analytical overview of indications, techniques, and outcomes of this technique in contemporary practice.

\section{Patients and Methods}

\section{Study design}

Data of patients who had undergone a minilaparoscopic urologic procedure at nine European institutions between 2009 and 2012 were retrospectively collected and gathered into a standardized datasheet. All patients had consented specifically and each group had performed the procedures according to its own protocols, inclusion criteria, and techniques.

\section{Outcome analysis}

Demographic data included age, gender, body mass index (BMI), past history of previous abdominal/pelvic surgery, the American Society of Anesthesiologists (ASA) score, and indications for ML.

Surgical procedures were classified as upper or lower urinary tract and as ablative or extirpative and reconstructive. The main surgical outcome parameters were analyzed, including operative time, estimated blood loss, perioperative complications, transfusions, length of hospital stay, and vi-

AU5 sual analog pain score (VAS). Intraoperative complications were classified according to a modified Satava system, ${ }^{13}$ whereas postoperative complications were graded according to Clavien-Dindo. ${ }^{14}$

Relevant operative data related to the surgical technique were recorded, including the number and size of the trocars as well as the approach (transperitoneal or retroperitoneal).

All patient data were collected in an excel spreadsheet (Microsoft Corp., Redmond, WA). Data of continuous variables are expressed as mean \pm standard deviation. Binary and categorical variables are reported as counts and percentages.

\section{Results}

\section{Demographics}

Overall, 192 patients (mean age $45.25 \pm 17.8$ years) were included in the analysis (Table 1). Most of them were nonobese (mean BMI $24.7 \pm 3.6 \mathrm{~kg} / \mathrm{m}^{2}$ ) at low estimated surgical risk (mean ASA 1.69 \pm 0.68 ). Indications for surgery were mostly nononcologic (132 cases, $68.8 \%$ ). Most of the procedures were done in the upper urinary tract (133 cases, $69.2 \%)$ and were mostly with a reconstructive intent (109 cases, $56.7 \%$ ).

\section{Instruments and surgical techniques}

In all cases the 3-mm minilaparoscopic set from Karl Storz $^{\circledR}$ (Tuttlingen, Germany) was used. A full range of instruments are available, including graspers, dissectors, scissors, suction-irrigation cannulas, and needle holders. This is a completely reusable set with $36-\mathrm{cm}$-long instruments, as well as shorter (20 and $30 \mathrm{~cm}$ long) 3 - and 2-mm instruments. The trocars are rigid cannulas with a metal conical-tipped trocar and silicone leaflet valves to help in maintaining pneumoperitoneum (Fig. 1). A variety of port configurations and combinations were adopted depending on the procedure.

For radical prostatectomy, the patient was placed in the Trendelenburg supine position (Fig. 2a). A 15-mm skin incision was made laterally to the umbilical scar to create a preperitoneal space by introducing a dissecting balloon trocar. After that, the balloon trocar was replaced by a $12-\mathrm{mm}$ optical trocar. Two 3.5-mm trocars were positioned medially to the anterior superior iliac spine, bilaterally. One 5-mm trocar was placed $\sim 3$ fingerbreadths medially to the left anterior superior iliac spine, whereas another $3.5-\mathrm{mm}$ trocar in the right pararectal line between the optical trocar and the previously placed right $3.5-\mathrm{mm}$ trocar.

For the pyeloplasty, both the transperitoneal ( 72 cases, $68.3 \%$ ) and retroperitoneal approaches (34 cases, 31.7\%) were used. The Anderson-Hynes technique was mostly adopted (85 cases, $79.5 \%$ ), whereas Foley Y-V (20 cases, $18.7 \%$ ) or Fenger (2 cases, $1.8 \%$ ) techniques were selectively used. For the transperitoneal approach (Fig. 2b), the first 3.5$\mathrm{mm}$ camera port was placed $2 \mathrm{~cm}$ laterally to the umbilicus, on the basis of patient body habitus. Then, under direct vision, other two $3.5-\mathrm{mm}$ trocars were placed along the midclavicular line, right or left depending on the side of the procedure. A fourth $3.5-\mathrm{mm}$ trocar, placed just below the xiphoid, was used as a liver retractor in case of right-sided procedures. ${ }^{10}$ For the retroperitoneal approach (Fig. 2c), a retroperitoneal tunnel was created through a $6 \mathrm{~mm}$ skin incision below the inferior edge of the 12th rib. To develop the retroperitoneal space, a 6-mm homemade dissecting balloon trocar was used. Then, two $3.5-\mathrm{mm}$ working trocars were placed under endoscopic control to achieve triangulation. ${ }^{11}$

For the nephrectomy, a transperitoneal approach was chosen for all the cases (Fig. 2d). A 5-mm optical trocar was introduced under direct vision through the umbilicus. ${ }^{12}$ Three to four $3.5-\mathrm{mm}$ trocars were then introduced under direct vision along the left pararectal line in a linear fashion. The camera port was switched to a 3-mm laparoscope and introduced through the most cephalic $3.5-\mathrm{mm}$ trocar. Finally, the fourth 3-mm trocar was normally placed at the ancillary line at the tip of the 12th rib to optimize retraction.

For the adrenalectomy, a lateral retroperitoneal approach was adopted in all the cases. Immediately below the 12th rib, a dissecting balloon trocar was inserted at the level of the inferior lumbar (Petit) triangle to develop a retroperitoneal space. After removal of the balloon trocar, a $3.5-\mathrm{mm}$ optical trocar was inserted and fixed with a silk suture. Under laparoscopic control, remaining ports were 
Table 1. Overall Cohort Demographic Data

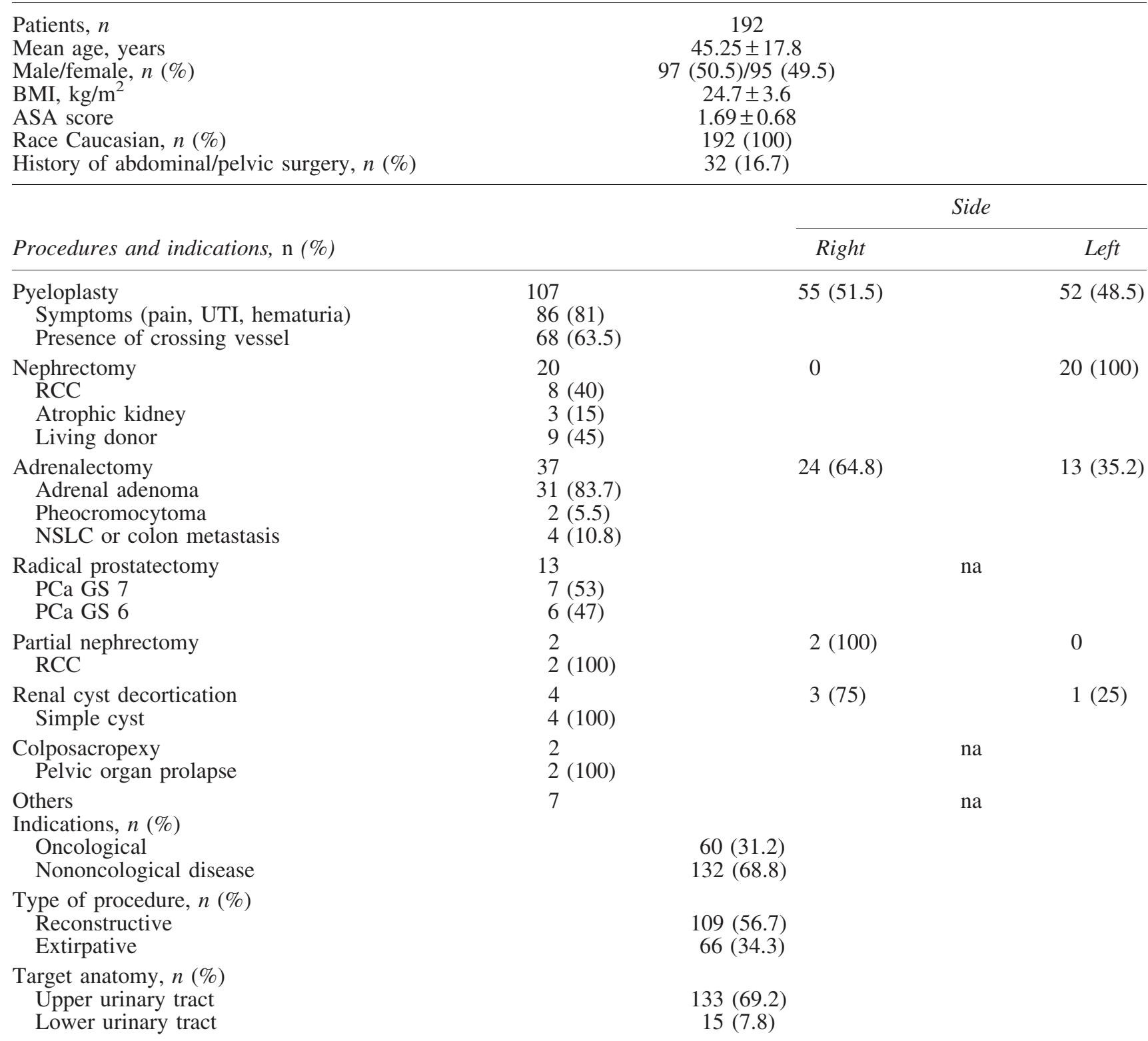

Data are expressed as mean $\pm(\mathrm{SD})$ or as counts and (percentage).

ASA, American Society of Anesthesiologists; BMI, body mass index; NSLC, nonsmall-cell lung cancer; UTI, .

introduced, including two $3.5-\mathrm{mm}$ trocars inserted below (along the lateral border of the sacrospinalis muscle) and anteriorly (along the anterior axillary line) to the 12th rib, respectively, so that the three ports formed a line corresponding to a subcostal incision.

\section{Surgical outcomes}

The overall operative time was $132.7 \pm 52.3$ minutes with an estimated blood loss of $60.9 \pm 47.6 \mathrm{~mL}$ while the mean hospital stay was $5 \pm 2.1$ days. In Table 2 the specific outcomes for the most commonly performed procedures are detailed.

A limited number of perioperative adverse events were observed in the present series. Only Satava grade 1 intra- operative complications were recorded $(8.3 \%$ of all the cases). These were represented by substitution of a 3-mm trocar with 5-mm or two 3-mm trocars with 5-mm as well as unplanned additions of extra 3-mm ports or 5-mm ports.

Overall, 58 postoperative complications were observed, accounting for a $30.2 \%$ complication rate. Most of them were Clavien grade $1(n=36 ; 18.7 \%$; fever, UTI, and respiratory infections), with only one $(0.5 \%)$ grade 3 and one $(0.5 \%)$ grade 4 complication recorded.

\section{Discussion}

Herein we reported the largest contemporary series of minilaparoscopic procedures in urology, detailing indications, outcomes, and technical nuances. 
FIG. 1. Storz ${ }^{\circledR}$ minilaparoscopy instruments: (A) 3.9$\mathrm{mm}$ trocars (compared with 5- and 10-mm ones); (B) 3$\mathrm{mm}$ instruments introduced through the $3.9-\mathrm{mm}$ trocar; (C) tip of bipolar 3-mm forceps; and (D) 2-mm instruments (compared with the 3$\mathrm{mm}$ ones).
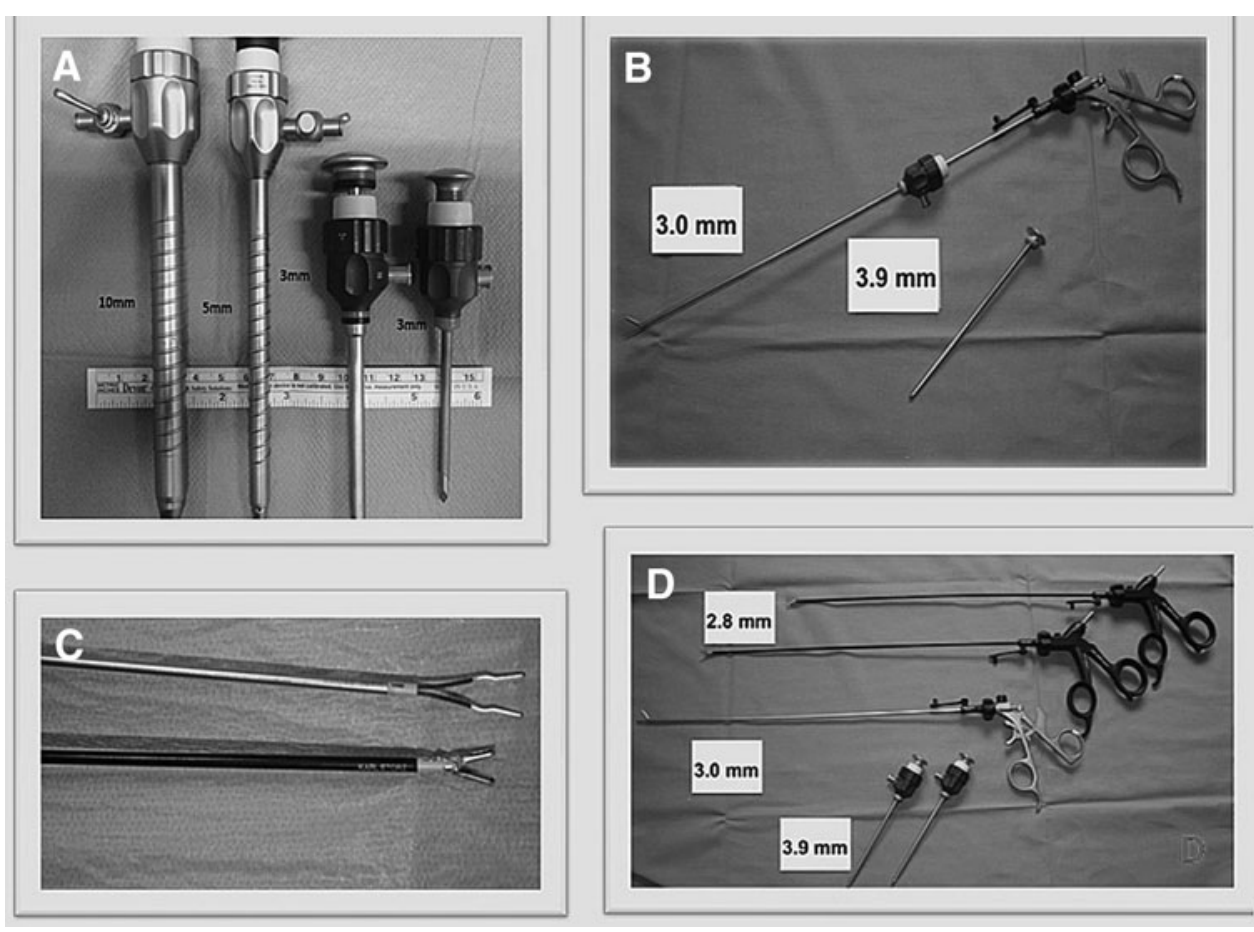

The idea of further reducing the minimal invasiveness of standard laparoscopy is not new. In the early 1990s this was proposed and experimented in different surgical specialties, ${ }^{15}$ including urology. ${ }^{16}$ However, it did not become popular because instruments were deemed to be too flimsy, reliable scopes were not available, and the surgical techniques themselves were not optimized. Ultimately, at that time ML seemed to have no significant advantages and did not progress as initially imagined.

In parallel with the recent development of potentially "scarless" surgical techniques, such as NOTES and LESS, there has been a renewed interest of the surgical community toward a rediscovery of ML. This interest has been driven by two main reasons: the boosting of manufacturers that leads to
FIG. 2. Port configuration for different minilaparoscopic procedures: (a) radical prostatectomy; (b) transperitoneal pyeloplasty; (c) retroperitoneoscopic pyeloplasty; and (d) nephrectomy.
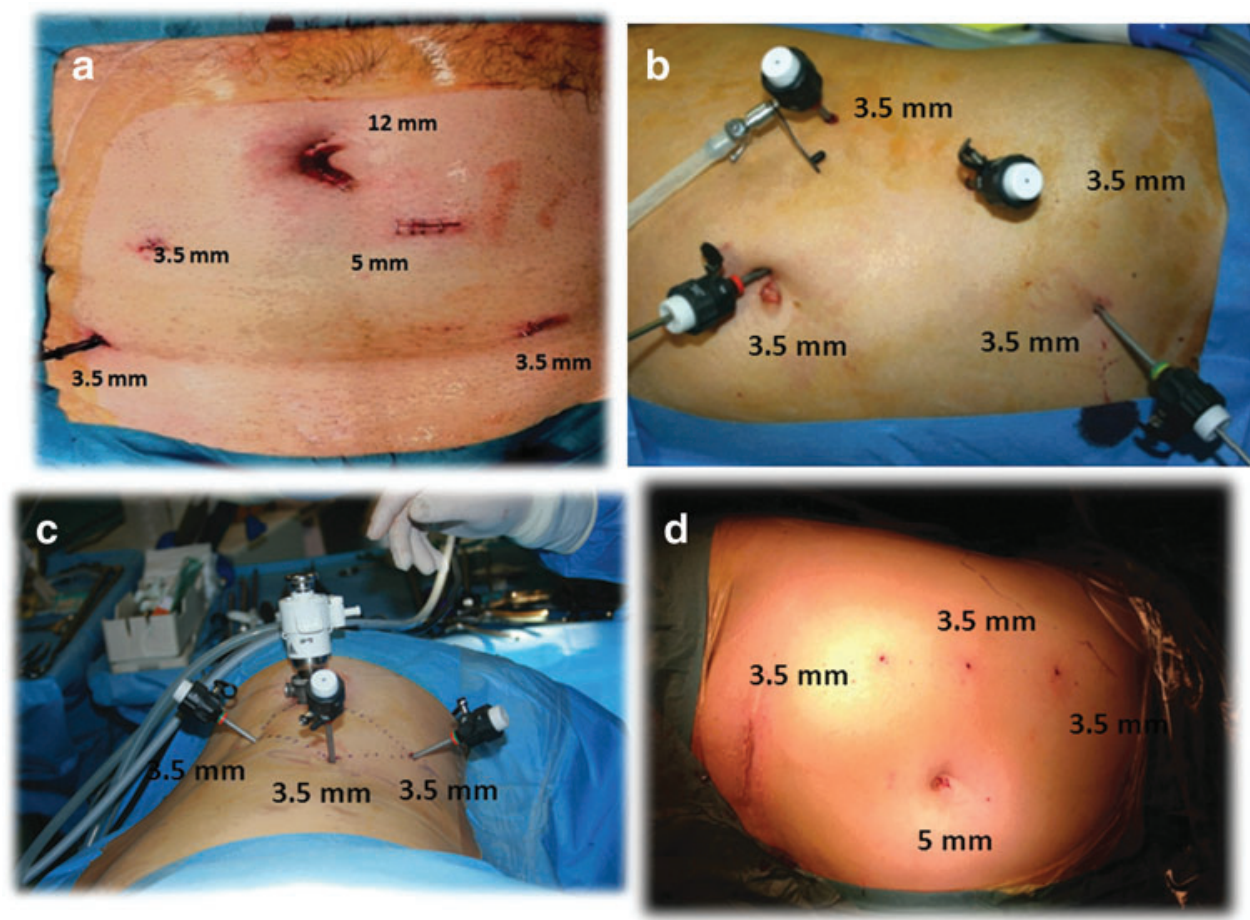
Table 2. Surgical Outcomes for Most Frequently Performed Mini Laparoscopic Procedures ${ }^{\mathrm{a}}$

\begin{tabular}{lccccc}
\hline & Case, $\mathrm{n}$ & ORT, min & EBL, $m L$ & LOS, days & VAS \\
\hline Pyeloplasty $^{\mathrm{b}}$ & 107 & $151.27 \pm 52$ & $55.42 \pm 47.8$ & $5.52 \pm 2.49$ & $0.59 \pm 0.79$ \\
Nephrectomy $^{\mathrm{c}}$ & 20 & $122.11 \pm 41.7$ & $52.50 \pm 43.27$ & $4.60 \pm 2.11$ & $1.10 \pm 0.57$ \\
Adrenalectomy $^{\mathrm{c}}$ & 37 & $102.78 \pm 32.73$ & $81.89 \pm 38.7$ & $4.75 \pm 0.81$ & $0.86 \pm 0.76$ \\
Radical prostatectomy & 13 & $114.23 \pm 21.20$ & $150.00 \pm 32.3$ & $3.69 \pm 1.11$ & $1.00 \pm 0.21$ \\
\hline
\end{tabular}

Values expressed as mean $\pm \mathrm{SD}$.

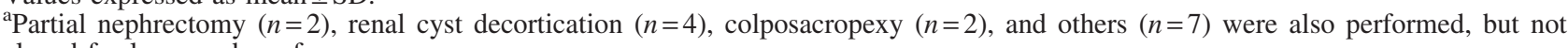
analyzed for low number of cases.

${ }^{\mathrm{b}}$ Including living donor $(n=9)$.

'Including partial $(n=8)$.

EBL, estimated blood loss; LOS, length of stay; ORT, operative room time; PCa GS, ; VAS, visual analog score at discharge.

the availability of a new generation of purpose-built instrumentation, ${ }^{17}$ and the fact that ML seems to be ready for immediate implementation as it is based on the same established principles of standard laparoscopy. ${ }^{18}$

At present, many procedures have been performed and in some cases the cosmetic benefit has also been demonstrated with an objective assessment. ${ }^{7}$ In urology, however, small case series and case-control studies have been reported so far. ${ }^{10-12}$ To our knowledge, this is the first large cohort reporting the outcomes of contemporary ML and providing an overview of the current applications in our surgical specialty.

When looking at the overall population of our study, patients were relatively young, nonobese, and at low estimated surgical risk. Obese patients do not represent an optimal indication for ML as smaller diameter instrument shafts bend more easily; on the other side, patients with multiple adhesions from previous surgery are less suitable too.

A large spectrum of the common urologic procedures for both upper and lower urinary tract diseases have been performed and shown to be feasible duplicating the principles of standard laparoscopy. Not surprisingly, reconstructive procedures, which do not require an additional incision to extract a surgical specimen, thus maximizing the benefits of the minilaparoscopic approach, were the most common.

Pyeloplasty represented the most common indication and was performed by using both transperitoneal and retroperitoneal approaches. Although the present series is devoid of data about patient satisfaction and scar assessment, Fiori and colleagues recently evaluated cosmetic outcomes in patients undergoing minilaparoscopic and standard pyeloplasty, by administering 3 months postoperatively a standardized questionnaire. ${ }^{10}$ The authors found that patients who underwent the minilaparoscopic approach were significantly more satisfied with their cosmetic results than those who received standard laparoscopic pyeloplasty. Besides the comparison between ML and standard laparoscopy, it also remains to be determined the comparison between ML and LESS. In a recent randomized trial on cholecystectomy, Lee and colleagues found LESS to be superior to ML in terms of cosmetic outcome, but not about postoperative pain and analgesic consumption. ${ }^{9}$

In a recent Cochrane meta-analysis of 13 trials comparing miniport versus standard port laparoscopic cholecystectomy, Gurusamy and colleagues observed that patients, in whom elective miniport laparoscopic cholecystectomy was completed successfully, had lower pain. ${ }^{19}$ These findings corroborate our finding of a very low VAS recorded at discharge.

Extirpative surgeries were also performed in our series. However, for radical prostatectomy, radical nephrectomy, and adrenalectomy, a liberal use of standard (larger) trocars and instruments is more likely. This can be explained by the need of overcoming the current shortcoming of minilaparoscopic instrumentation and minimizing the surgical risk. Despite technological improvements obtained with the latest generation of instruments, some drawbacks are still present. ${ }^{19}$ The quality of vision provided by the 3-mm scope is inferior in terms of image resolution, clarity, and light transmitting capacity, in comparison to a 10-mm laparoscope. Moreover, in case of bleeding, the illumination-induced light absorption causes a substantial decrease in image quality. Clip applicators are unavailable, and this is a clear limitation, in particular, when an extirpative procedure is planned. The use of bipolar can partially compensate for this shortcoming. The suction irrigation cannula, due to its small diameter, has poor flow characteristics and, in some cases, fails to maintain a bloodless field. The evacuation of smoke can also be compromised by the small caliber ports, especially when an instrument is inserted.

Nomenclature for ML, as well as other scarless techniques, has been recently summarized by Georgiou and colleagues. ${ }^{20}$ According to this nomenclature, minilaparoscopy implies the use of 3- to 5-mm rigid instruments and telescopes, whereas hybrid minilaparoscopy implies the use of larger ports (10 mm).

Similar to what has been done for standard laparoscopy ${ }^{21}$ and for LESS, ${ }^{22} \mathrm{ML}$ as well must be scrutinized for the risk of perioperative adverse events. Adopting two standardized reporting systems, the Satava and the Clavien Dindo, our data suggested that ML is overall a safe technique, despite this analysis, including the early experience of participating centers. Intraoperative adverse events only included the replacement of 3-mm trocars by $5-\mathrm{mm}$ trocars or the addition of extra ports (Satava grade 1), whereas no conversion to open surgery was needed. The overall rate of those events was $8.3 \%$. Postoperative adverse events were recorded in $30.2 \%$ of cases, most of them being low Clavien grade (1 and 2).

When considering main perioperative outcomes, it can be grossly estimated that some of the most commonly performed procedures in the present series (e.g., pyeloplasty, nephrectomy, adrenalectomy, and radical prostatectomy) compare 
favorably with the reported series of their laparoscopic counterparts. ${ }^{23-26}$

Some important limitations of the present study need to be mentioned. First, it is a retrospective analysis of prospectively collected data, so that the assessment has been limited to variables that were available with sufficient quality only. A control group was not considered and the follow-up is short. Moreover, the surgical procedures were performed by surgical teams with previous extensive laparoscopic background, so that transferring these findings in a different setting should be done cautiously.

\section{Conclusions}

This study provides an overview of the recent evolution of urologic ML in multiple European laparoscopic expertise centers. A broad range of common procedures can be safely and effectively performed with this newly rediscovered technique, given the current availability of purpose-built instrumentation. By duplicating the principles of standard laparoscopy, but potentially offering less surgical scar and trauma, ML can be regarded as a viable option when looking for a virtually scarless surgery.

\section{Disclosure Statement}

No competing financial interests exist.

\section{References}

1. Autorino R, Cadeddu JA, Desai MM, et al. Laparoendoscopic single-site and natural orifice transluminal endoscopic surgery in urology: A critical analysis of the literature. Eur Urol 2011;59:26-45.

2. Alcaraz A, Musquera M, Peri L, et al. Feasibility of transvaginal natural orifice transluminal endoscopic surgeryassisted living donor nephrectomy: Is kidney vaginal delivery the approach of the future? Eur Urol 2011;59: 1019-1025

3. Porpiglia F, Fiori C, Morra I, Scarpa RM. Transvaginal natural orifice transluminal endoscopic surgery-assisted minilaparoscopic nephrectomy: A step towards scarless surgery. Eur Urol 2011;60:862-866.

4. Kaouk JH, Autorino R, Kim FJ, et al. Laparoendoscopic single-site surgery in urology: Worldwide multi-institutional analysis of 1076 cases. Eur Urol 2011;60:998-1005.

5. Pfluke JM, Parker M, Stauffer JA, et al. Laparoscopic surgery performed through a single incision: A systematic review of the current literature. J Am Coll Surg 2011;212:113-118.

6. Liatsikos E, Kyriazis I, Kallidonis P, Do M, Dietel A, Stolzenburg JU. Pure single-port laparoscopic surgery or mix of techniques? World J Urol 2012;30:581-587.

7. Pini G, Porpiglia F, Micali S, Rassweiler J. Minilaparoscopy, needlescopy and microlaparoscopy: Decreasing invasiveness, maintaining the standard laparoscopic approach. Arch Esp Urol 2012;65:366-383.

8. Ghezzi F, Cromi A, Siesto G, Uccella S, Boni L, Serati M, Bolis P. Minilaparoscopic versus conventional laparoscopic hysterectomy: Results of a randomized trial. J Minim Invasive Gynecol 2011;18:455-461.

9. Lee PC, Lo C, Lai PS, Chang JJ, Huang SJ, Lin MT, Lee $\mathrm{PH}$. Randomized clinical trial of single-incision laparoscopic cholecystectomy versus minilaparoscopic cholecystectomy. Br J Surg 2010;97:1007-1012.
10. Fiori C, Morra I, Bertolo R, Mele F, Chiarissi ML, Porpiglia F. Standard vs mini-laparoscopic pyeloplasty: Perioperative outcomes and cosmetic results. BJU Int 2013;111(3 Pt B): E121-E126.

11. Pini G, Goezen AS, Schulze M, Hruza M, Klein J, Rassweiler JJ. Small-incision access retroperitoneoscopic technique (SMART) pyeloplasty in adult patients: Comparison of cosmetic and post-operative pain outcomes in a matchedpair analysis with standard retroperitoneoscopy: Preliminary report. World J Urol 2012;30:605-611.

12. Breda A, Villamizar JM, Faba OR, Caliolo C, de Gracia A, Gausa L, de Leon JP, Villavicencio H. Laparoscopic live donor nephrectomy with the use of 3-mm Instruments and laparoscope: Initial experience at a tertiary center. Eur Urol 2012;61:840-844.

13. Kazaryan AM, Røsok BI, Edwin B. Morbidity assessment in surgery: Refinement proposal based on a concept of perioperative adverse events. ISRN Surg 2013;2013: 625093.

14. Dindo D, Demartines N, Clavien PA. Classification of surgical complications: A new proposal with evaluation in a cohort of 6336 patients and results of a survey. Ann Surg 2004;240:205-213.

15. Carvalho GL, Loureiro MP, Bonin EA. Renaissance of Minilaparoscopy in the NOTES and single port era. JSLS 2011;15:585-588.

16. Soble JJ, Gill IS. Needlescopic urology: Incorporating 2-mm instruments in laparoscopic surgery. Urology 1998;52: 187-194.

17. Krpata DM, Ponsky TA. Needlescopic surgery: What's in the toolbox? Surg Endosc 2013;27:1040-1044.

18. Autorino R, Kim FJ, Rassweiler J, et al. Mini-laparoscopy, laparoendoscopic single-site surgery and natural orifice transluminal endoscopic surgery-assisted laparoscopy: Novice surgeons' performance and perception in a porcine nephrectomy model. BJU Int 2012;110(11 Pt C):E991-E996.

19. Pini G, Rassweiler J. Minilaparoscopy and laparoendoscopic single-site surgery: Mini- and single-scar in urology. Minim Invasive Ther Allied Technol 2012;21:8-25.

20. Georgiou AN, Rassweiler J, Herrmann TR, et al. Evolution and simplified terminology of natural orifice transluminal endoscopic surgery (NOTES), laparoendoscopic single-site surgery (LESS), and mini-laparoscopy (ML). World J Urol 2012;30:573-580

21. Permpongkosol S, Link RE, Su LM, et al. Complications of 2,775 urological laparoscopic procedures: 1993 to 2005. J Urol 2007;177:580.

22. Autorino R, Kaouk JH, Yakoubi R, et al. Urological laparoendoscopic single site surgery: Multi-institutional analysis of risk factors for conversion and postoperative complications. J Urol 2012;187:1989-1994.

23. Autorino R, Eden C, El-Ghoneimi A, Guazzoni G, Buffi N, Peters CA, Stein RJ, Gettman M. Robot-assisted and laparoscopic repair of ureteropelvic junction obstruction: A systematic review and meta-analysis. Eur Urol 2013; 65:430-452.

24. Mues AC, Haramis G, Rothberg MB, Okhunov Z, Casazza C, Landman J. Contemporary experience with laparoscopic radical nephrectomy. J Laparoendosc Adv Surg Tech A 2011;21:15-18.

25. Zacharias M, Haese A, Jurczok A, Stolzenburg J-U, Fornara P. Transperitoneal laparoscopic adrenalectomy: Outline of the preoperative management, surgical approach, and outcome. Eur Urol 2006;49:448-459. 
26. Kowalczyk KJ, Yu HY, Ulmer W, Williams SB, Hu JC. Outcomes assessment in men undergoing open retropubic radical prostatectomy, laparoscopic radical prostatectomy, and robotic-assisted radical prostatectomy. World J Urol 2012;30:85-89.

Address correspondence to: Riccardo Autorino, MD, PhD, FEBU Center for Laparoscopic and Robotic Surgery Glickman Urological and Kidney Institute Cleveland Clinic 9500 Euclid Avenue/Q-10 Cleveland, $\mathrm{OH} 44195$

E-mail: autorir@ccf.org; ricautor@gmail.com

\section{Abbreviations Used}

$\mathrm{ASA}=$ American Society of Anesthesiologists

$\mathrm{BMI}=$ body mass index

$\mathrm{EBL}=$ estimated blood loss

LESS $=$ laparoendoscopic single-site surgery

LOS $=$ length of stay

$\mathrm{ML}=$ minilaparoscopy

NOTES $=$ natural orifice translumenal endoscopic surgery

$\mathrm{NSLC}=$ nonsmall-cell lung cancer

ORT $=$ operative room time

$\mathrm{PCa} \mathrm{GS}=$

$\mathrm{SD}=$ standard deviation

$\mathrm{UTI}=$

$\mathrm{VAS}=$ 


\section{AUTHOR QUERY FOR END-2014-0134-VER9-PORPIGLIA_1P}

AU1: Please note that gene symbols in any article should be formatted as per the gene nomenclature. Thus, please make sure that gene symbols, if present in this article, are italicized.

AU2: Please review all authors' surnames for accurate indexing citations.

AU3: Please mention the authors' degrees.

AU4: Please confirm the correctness of authors' affiliations.

AU5: Please fix the expansion of the acronym VAS: "visual analog pain score" or "visual analog score at discharge"?

AU6: Gurusamy and colleagues do not match with Ref. (20). Please check.

AU7: Ref. 19 has been deleted as it was a duplicate of Ref. 9, and Ref. citations in the text have been renumbered accordingly. Please check.

AU8: Please define PCa GS and UTI. 